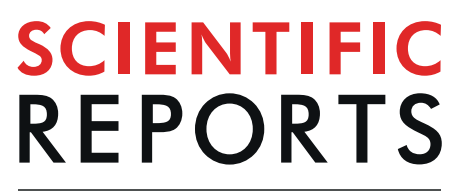

natureresearch

\title{
Novel patient risk factors and validation of a difficulty scoring system in laparoscopic repeat hepatectomy
}

\author{
Yukiyasu Okamura $\mathbb{D}^{*}$, Yusuke Yamamoto, Teiichi Sugiura, Takaaki Ito, Ryo Ashida, \\ Katsuhisa Ohgi \& Katsuhiko Uesaka
}

The indications for laparoscopic liver resection have expanded; however, the safety and benefits of laparoscopic repeat hepatectomy $(\mathrm{LRH})$ remain unclear. We retrospectively reviewed data from 137 patients who underwent partial hepatectomy or left lateral sectionectomy without thoracotomy. We compared patients' clinical factors using a difficulty scoring system for $\mathrm{LRH}$. We defined factors associated with blood loss volumes in the 75th percentile or above as risk factors for bleeding in open repeat hepatectomy, and determined whether these factors were useful for $\mathrm{LRH}$ risk assessment. Open repeat hepatectomy and LRH was performed in 96 and 41 patients, respectively. Four of 41 (9.8\%) patients undergoing LRH were converted to laparotomy. Blood loss volume was significantly greater in the intermediate-risk group than in the low-risk group $(P=0.046)$. Multivariate analysis revealed that the presence of tumours located adjacent and caudal or dorsal to the primary tumour site was an independent risk factor for bleeding in LRH (odds ratio 3.21, 95\% confidence interval 1.16-8.88, $P=0.024)$. Our study validated the usefulness of a difficulty scoring system, identified patient factors that predicted the difficulty of $\mathrm{LRH}$, and presented a novel difficulty scoring system for $\mathrm{LRH}$ based on an existing difficulty scoring system.

Repeat hepatectomy is an effective treatment for liver tumours, including hepatocellular carcinoma (HCC) $)^{1,2}$ and colorectal metastatic tumours ${ }^{3,4}$. However, repeat hepatectomy is generally more technically difficult than primary hepatectomy because of adhesions and anatomical changes caused by the previous resection.

Laparoscopic techniques and instruments have progressed over the last two decades, and laparoscopic liver resection (LLR) is now frequently performed to treat liver malignant tumours worldwide. The National Clinical Database in Japan demonstrated that LLR is associated with less blood loss, fewer postoperative complications, and a shorter duration of postoperative hospitalization than conventional open hepatectomy ${ }^{5}$. As a result, the indications for LLR have been expanded, and laparoscopic repeat hepatectomy (LRH) has been introduced in several institutions. However, the safety and outcomes of LRH have not been fully assessed, as only a small number of studies with small sample sizes have been published ${ }^{6-10}$.

Ban et al. ${ }^{11}$ introduced a difficulty scoring system to assess the difficulty of LLR, and some studies have validated this scoring system ${ }^{12-15}$. However, it is difficult to use the scoring system to evaluate the difficulty of LRH, as the system does not assess factors related to repeat hepatectomy.

To identify the factors affecting intraoperative blood loss in LRH, we retrospectively analysed data from patients undergoing open repeat hepatectomy and evaluated whether the patient factors indicative of a high risk of blood loss in open repeat hepatectomy were useful for risk assessment in LRH. We also validated the utility of the difficulty scoring system proposed by Ban et al. ${ }^{11}$, modified the system to include our novel patient-related risk factors, and then evaluated the modified system for assessing the risk of bleeding in patients undergoing LRH.

\section{Results}

Patient characteristics. Among the 137 patients who underwent partial hepatectomy or left lateral sectionectomy without thoracotomy, 41 patients underwent LRH, while 96 underwent open repeat hepatectomy (Table 1). Four patients undergoing LRH (9.8\%) were converted to laparotomy because the target tumour could 


\begin{tabular}{|c|c|c|c|}
\hline & LRH & Open-Re-Hx & \\
\hline & $\mathrm{n}=41$ & $\mathrm{n}=96$ & $P$ \\
\hline Age (years) $)^{\#}$ & $65(25-84)$ & $67(37-84)$ & 0.851 \\
\hline Gender (male/female) & $27 / 14$ & $66 / 30$ & 0.842 \\
\hline ASA-PS (3) & $4(9.8)$ & $7(7.3)$ & 1.000 \\
\hline Tumour type (HCC/metastatic tumour) & $19 / 22$ & $27 / 69$ & 0.049 \\
\hline Type of approach to the previous hepatectomy (Lap-Hx) & $15(36.6)$ & $4(4.2)$ & $<0.001$ \\
\hline Number of the previous hepatectomy ( 3 or more) & $6(14.6)$ & $31(32.3)$ & 0.037 \\
\hline Pringle manoeuvre (performed) & $17(41.5)$ & $52(54.2)$ & 0.195 \\
\hline Time of Pringle manoeuvre (minutes) ${ }^{\#}$ & $0(0-133)$ & $16(0-216)$ & 0.374 \\
\hline Year of surgery (2015-2018) & $37(90.2)$ & $41(42.7)$ & $<0.001$ \\
\hline Tumour diameter $(\mathrm{mm})^{\#}$ & $15.5(4.0-40)$ & $20.0(8.0-53)$ & 0.035 \\
\hline Tumour number (multiple) & $5(12.2)$ & $24(25.0)$ & 0.113 \\
\hline Tumour location (S1, S7 or S8) & $13(31.7)$ & $51(53.1)$ & 0.025 \\
\hline Tumours located in the ipsilateral of the previous surgical site & $19(46.3)$ & $75(78.9)$ & 0.001 \\
\hline $\begin{array}{l}\text { Tumours located in the adjacent area on the cranial or dorsal } \\
\text { side of the previous surgical site }\end{array}$ & $14(34.1)$ & $47(49.0)$ & 0.134 \\
\hline Intraoperative blood loss $(\mathrm{mL})^{\#}$ & $\begin{array}{l}60 \text { (small } \\
\text { amount-3,260) }\end{array}$ & $\begin{array}{l}311 \text { (small } \\
\text { amount-2,565) }\end{array}$ & $<0.001$ \\
\hline Operation time (minutes) ${ }^{\#}$ & $178(72-368)$ & $203(64-767)$ & 0.099 \\
\hline Morbidity $\geq$ Clavien-Dindo grade $3 a$ & $1(2.4)$ & $6(6.3)$ & 0.674 \\
\hline R0 resection & 36 of $39(92.3)$ & 91 of $93(97.8)$ & 0.153 \\
\hline Surgical margin $(\mathrm{mm})^{\#}$ & $4.5(0-25)$ & $3(0-30)$ & 0.066 \\
\hline Postoperative hospital stay (days) ${ }^{\#}$ & $5(4-41)$ & $9(4-35)$ & $<0.001$ \\
\hline Cirrhosis (present) & $4(9.8)$ & $7(7.3)$ & 0.733 \\
\hline
\end{tabular}

Table 1. Comparison of the clinicopathological characteristics of the laparoscopic repeat hepatectomy group versus the open repeat hepatectomy group LRH, laparoscopic repeat hepatectomy; Open-Re-Hx, open repeat hepatectomy; ASA-PS, American Society of Anesthesiologists physical status; HCC, hepatocellular carcinoma; Lap-Hx, laparoscopic hepatectomy; S, segment. Values in parentheses are percentages unless indicated otherwise $^{\#}$.value is expressed as the median (range).

not be detected under laparoscopic surgery $(\mathrm{N}=2)$, severe adhesions were present $(\mathrm{N}=1)$, or haemorrhage occurred $(\mathrm{N}=1)$. Repeat hepatectomy was performed in 46 patients with HCC and 91 patients with metastatic tumours. HCC was significantly more frequently treated via LRH than via open repeat hepatectomy (46.3\% vs. $28.1 \%$, respectively; $P=0.049$ ). Compared with the patients who underwent open repeat hepatectomy, the patients who underwent LRH had a significantly lower incidence of tumours located in segments 1, 7, or $8(31.7 \%$ vs. $53.1 \%$, respectively; $P=0.025)$ and a significantly lower incidence of tumours located ipsilateral to the previous hepatectomy site ( $46.3 \%$ vs. $78.9 \%$, respectively; $P=0.001)$. Among the 41 patients who underwent LRH, 37 (90.2\%) underwent surgery later in the study period (2015-2018).

Compared with patients who underwent open repeat hepatectomy, patients who underwent LRH had a significantly smaller median volume of intraoperative blood loss $(60 \mathrm{~mL}$ vs. $311 \mathrm{~mL}, P<0.001)$ and a significantly shorter median postoperative hospital stay ( 5 days vs. 9 days, $P<0.001$ ).

Assessment of the usefulness of the Iwate difficulty scoring system in LRH. The Iwate difficulty scoring system was used to classify 15 and 25 patients who underwent LRH into the intermediate- and low-risk groups, respectively, (Table 2) ${ }^{11}$. A difficulty index value was not obtained in one patient with a tumour located in segment 1 . There was no high-risk group with a score $\geq 7$ because major hepatectomy is not performed via laparoscopic surgery in our institution.

Compared with the low-risk group, the intermediate-risk group had a significantly higher incidence of tumours located in segments 1,7 , or $8(P<0.001)$, significantly higher incidence of tumours ipsilateral to the previous surgical site $(P=0.003)$, and significantly higher incidence of tumours adjacent and cranial or dorsal to the previous surgical site $(P<0.001)$. The intermediate-risk group also had a significantly greater blood loss volume $(P=0.046)$ and longer duration of postoperative hospitalisation $(P=0.016)$ than the low-risk group.

Factors affecting massive blood loss in patients undergoing open repeat hepatectomy. The median intraoperative blood loss volume in the 96 patients who underwent open repeat hepatectomy was $311 \mathrm{~mL}$ (range: small immeasurable amount-2,565 mL), and the 75th percentile for intraoperative blood loss volume was $547 \mathrm{~mL}$ (Fig. 1). To identify the risk factors for massive intraoperative blood loss, we extracted factors associated with patients with a blood loss volume $\geq$ the 75 th percentile in open repeat hepatectomy. Multivariate analysis revealed that the presence of tumours located adjacent and cranial or dorsal to the previous surgical site (odds ratio: 3.21 , 95\% confidence interval: $1.16-8.88, P=0.024)$ was an independent risk factor for massive intraoperative blood loss in open repeat hepatectomy (Table 3 ). 


\begin{tabular}{|c|c|c|c|}
\hline & $\begin{array}{l}\text { Intermediate (Difficulty } \\
\text { index } 4 \text { or more) }\end{array}$ & $\begin{array}{l}\text { Low (Difficulty } \\
\text { index less than } 4 \text { ) }\end{array}$ & \\
\hline & $\mathrm{n}=15$ & $\mathrm{n}=25$ & $P$ \\
\hline Age (years) $)^{\#}$ & $68(46-84)$ & $65(25-83)$ & 0.761 \\
\hline Gender (male/female) & $11 / 4$ & $15 / 10$ & 0.502 \\
\hline ASA-PS (3) & $1(6.7)$ & $3(12.0)$ & 1.000 \\
\hline Tumour type (HCC/metastatic tumour) & $7 / 8$ & $12 / 13$ & 1.000 \\
\hline Type of approach to the previous hepatectomy (Lap-Hx) & $7(46.7)$ & $7(28.0)$ & 0.310 \\
\hline Number of the previous hepatectomy ( 3 or more) & $4(26.7)$ & $2(8.0)$ & 0.174 \\
\hline Pringle manoeuvre (performed) & $8(53.3)$ & $9(36.0)$ & 0.336 \\
\hline Time of Pringle manoeuvre (minutes) $)^{\#}$ & $0(0-133)$ & $0(0-108)$ & 0.720 \\
\hline Year of surgery $(2015-2018)$ & $14(93.3)$ & $14(56.0)$ & 0.015 \\
\hline Tumour diameter $(\mathrm{mm})^{\#}$ & $20(7-38)$ & $15(4-40)$ & 0.041 \\
\hline Tumour number (multiple) & $3(20.0)$ & $2(8.0)$ & 0.345 \\
\hline Tumour location (S1, S7 or S8) & $12(80.0)$ & 0 & $<0.001$ \\
\hline Tumours located in the ipsilateral of the previous surgical site & $12(80.0)$ & $7(28.0)$ & 0.003 \\
\hline $\begin{array}{l}\text { Tumours located in the adjacent area on the cranial or dorsal } \\
\text { side of the previous surgical site }\end{array}$ & $11(73.3)$ & $3(12.0)$ & $<0.001$ \\
\hline Intraoperative blood loss $(\mathrm{mL})^{\#}$ & 125 (small amount-3,260) & $\begin{array}{l}50(\text { small } \\
\text { amount-784) }\end{array}$ & 0.046 \\
\hline Operation time (minutes) $)^{\#}$ & $218(83-368)$ & $174(72-355)$ & 0.083 \\
\hline Converted to laparotomy & $3(20.0)$ & $1(4.0)$ & 0.139 \\
\hline Morbidity $\geq$ Clavien-Dindo grade $3 a$ & $1(6.7)$ & 0 & 0.375 \\
\hline Postoperative hospital stay (days) & $6(5-41)$ & $5(4-17)$ & 0.016 \\
\hline
\end{tabular}

Table 2. Comparisons of clinical factors of the patients who underwent LRH who were classified as having an intermediate or low risk of massive intraoperative blood loss in accordance with the Iwate difficulty scoring system. LRH, laparoscopic repeat hepatectomy; ASA-PS, American Society of Anesthesiologists physical status; HCC, hepatocellular carcinoma; Lap-Hx, laparoscopic hepatectomy; S, segment. Values in parentheses are percentages unless indicated otherwise ${ }^{\#}$.value is expressed as the median (range).

Assessment of whether the risk factors for massive blood loss in open repeat hepatectomy are useful in predicting the difficulty of LRH. Among the 41 patients who underwent LRH, 14 patients $(34.1 \%)$ had tumours located adjacent and cranial or dorsal to the previous surgical site. The difficulty index in patients with this risk factor was significantly higher than that in patients without this risk factor (median: $5 \mathrm{vs.}$ 2.5 , respectively; $P=0.001$ ). The duration of the Pringle manoeuvre in patients with this risk factor was significantly longer than that in patients without this risk factor (median: 46 minutes vs. 0 minutes; $P=0.045$ ). Patients with this risk factor had a significantly greater blood loss volume (median: $284 \mathrm{~mL}$ vs. $50 \mathrm{~mL}, P=0.009$ ) and longer operation time (median: $253 \mathrm{~min}$. vs. $170 \mathrm{~min}, P=0.002$ ) than patients without this risk factor (Table 4). However, there was no significant difference between patients with and without this risk factor in the rate of conversion to laparotomy $(P=0.602)$.

Extent to which the presence of tumours located adjacent and cranial or dorsal to the previous surgical site affects the Iwate difficulty scoring system. We created a novel difficulty scoring system based on the Iwate difficulty scoring system to add the presence of tumours located adjacent and cranial to the previous surgical site as a risk assessment factor. Assuming that the effect of the risk factor (presence of tumours located adjacent and cranial or dorsal to the previous surgical site) on the difficulty of LRH was given a score of 3 , the distributions of the difficulty index scores in accordance with the Iwate and our novel difficulty scoring systems are shown in Fig. 2. When cut-off scores of $\geq 4,5,6$, and 7 were used to define the high-risk LRH group, this resulted in the distribution of 18 (43.9\%), 17 (41.5\%), 13 (32.5\%), and 11 (26.8\%) patients, respectively, into the high-risk LRH group, with respective $P$ values of $0.034,0.015,0.003$, and 0.009 for comparisons of intraoperative blood loss volume. Moreover, when the factor affecting the difficulty of LRH was given a score of 1,2 , or 4 , $P$ values of less than 0.003 were not obtained, regardless of where the cut-off point was set. Therefore, we defined the optimal cut-off value for the high-risk LRH group as 6 in the novel difficulty scoring system. However, the rate of conversion to laparotomy did not significantly change, regardless of where the cut-off point was set.

\section{Discussion}

The present study showed that the presence of tumours located adjacent and cranial or dorsal to the previous surgical site, which was a factor related to massive blood loss in open repeat hepatectomy, was also useful in assessing the difficulty of LRH. Furthermore, our novel difficulty scoring system based on the Iwate difficulty scoring system was useful for predicting massive blood loss in LRH.

While most studies show that LRH is associated with less blood loss, fewer postoperative complications, and a shorter duration of postoperative hospitalisation compared with conventional open hepatectomy ${ }^{6-10}$, to our knowledge, the present study is the first to evaluate the use of these factors to predict the difficulty of LRH. 
A

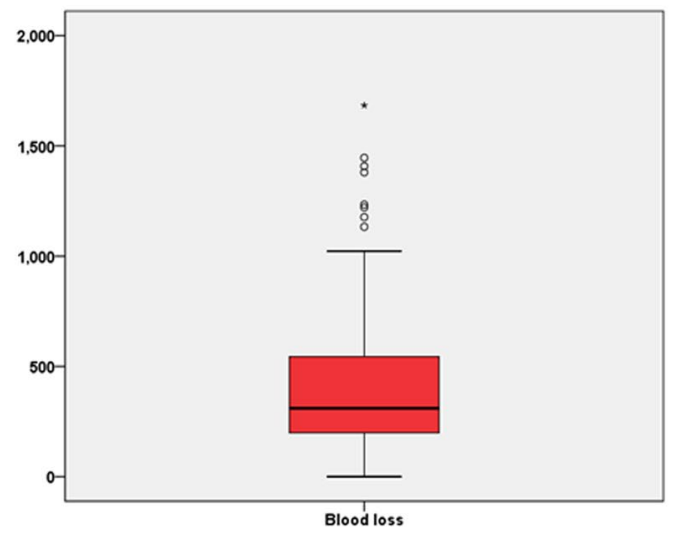

B

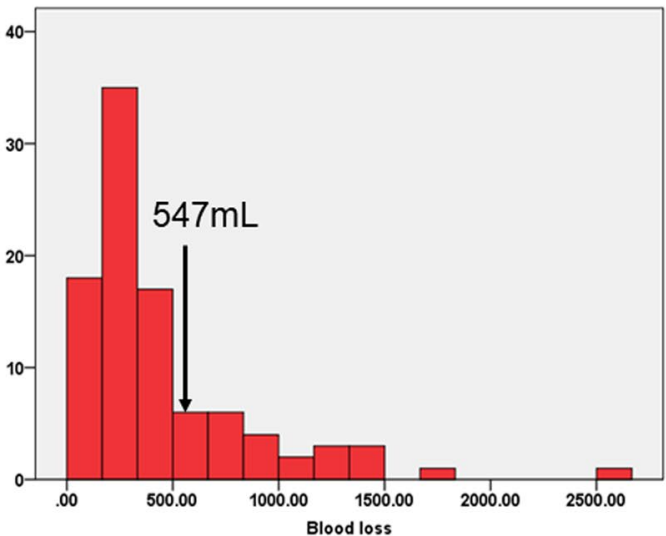

Figure 1. Bar graph showing intraoperative blood loss volume. (A) The $\geq 75$ th percentile for blood loss is indicated by the arrows $(\mathbf{B})$.

\begin{tabular}{|c|c|c|c|c|}
\hline \multirow[b]{2}{*}{ Variables } & \multicolumn{2}{|l|}{ Univariate } & \multicolumn{2}{|l|}{ Multivariate } \\
\hline & $\begin{array}{l}\text { Odds ratio ( } 95 \% \\
\text { confidence interval) }\end{array}$ & $P$ & $\begin{array}{l}\text { Odds ratio ( } 95 \% \\
\text { confidence interval) }\end{array}$ & $P$ \\
\hline \multicolumn{5}{|l|}{ Massive blood loss } \\
\hline Age ( $>67$ years $)$ & $0.54(0.21-1.38)$ & 0.198 & & \\
\hline Gender (male) & $1.50(0.53-4.27)$ & 0.447 & & \\
\hline ASA-PS (3) & $0.63(0.07-5.70)$ & 0.677 & & \\
\hline Tumour type (metastatic tumour) & Not obtained & 0.998 & & \\
\hline Number of the previous hepatectomy ( 3 or more) & $1.36(0.52-3.59)$ & 0.529 & & \\
\hline Year of surgery $(2015-2018)$ & $1.40(0.51-3.78)$ & 0.513 & & \\
\hline Tumour diameter $(>20 \mathrm{~mm})$ & $0.94(0.35-2.50)$ & 0.901 & & \\
\hline Tumour number (multiple) & $2.96(1.09-8.04)$ & 0.032 & & \\
\hline Tumour location (S1, S7 or S8) & $1.32(0.52-3.37)$ & 0.556 & & \\
\hline Tumours located in the ipsilateral of the previous surgical site (yes) & $3.94(0.85-18.4)$ & 0.081 & & \\
\hline $\begin{array}{l}\text { Tumours located in the adjacent area on the cranial or dorsal side of } \\
\text { the previous surgical site (yes) }\end{array}$ & $3.40(1.25-9.22)$ & 0.016 & $3.21(1.16-8.88)$ & 0.024 \\
\hline
\end{tabular}

Table 3. Predictors of massive intraoperative blood loss in open repeat hepatectomy. ASA-PS, American Society of Anesthesiologists physical status; S, segment.

Consistent with previous results ${ }^{6-10}$, our results showed that LRH resulted in less intraoperative blood loss and a shorter duration of postoperative hospitalisation compared with open repeat hepatectomy. In our study, we found that surgeons tended to avoid performing LRH in patients with tumours located in segments 1,7 or 8 and patients with tumours ipsilateral to the previous surgical site, as the difficulty of LRH may be high in these patients. Therefore, there is an urgent need for a difficulty scoring system for LRH.

Ban et al. ${ }^{11}$ proposed the Iwate difficulty scoring system to assess the difficulty of LLR in 2014 , and subsequent studies have validated this scoring system and confirmed its usefulness ${ }^{12-15}$. When the Iwate difficulty scoring system is used to classify patients into low-, intermediate-, and high-risk groups, the intraoperative blood loss volume and operation time reportedly significantly differ between the low- and high-risk groups and between the intermediate- and high-risk groups, but not between the low- and intermediate-risk groups ${ }^{11}$. However, the Iwate difficulty scoring system does not include factors related to repeat hepatectomy and tumours located in segment 1 , as the presence of tumours in segment 1 was considered a contraindication for LLH when this procedure was first introduced.

Five years have passed since Ban et al. ${ }^{11}$ published the Iwate difficulty scoring system. In that time, the indications for LLR have been expanded, and LRH has been introduced. Therefore, it is now important to determine how much weight should be assigned using the Iwate difficulty scoring system to the factors affecting patients undergoing repeat hepatectomy or patients with tumours located in segment 1 . In the current study, although no patients had a difficulty index of $\geq 7$, which corresponds to the high-risk group in the Iwate difficulty scoring system, the intraoperative blood loss volume significantly differed between the low- and intermediate-risk groups, which was not seen in the original study by Ban et al. ${ }^{11}$ In the original study, the intermediate-risk group was defined as having a difficulty index of 4-6, while the high-risk group was defined as having a difficulty index of $\geq 7^{11}$. Comparing our results with those of Ban et al. ${ }^{11}$, the factor "repeat hepatectomy" may be equivalent to 


\begin{tabular}{|c|c|c|c|}
\hline & $\begin{array}{l}\text { Tumours located in the adjacent } \\
\text { area on the cranial or dorsal } \\
\text { side of the primary site }\end{array}$ & $\begin{array}{l}\text { Tumours located in the adjacent } \\
\text { area on the caudal or ventral } \\
\text { side of the primary site }\end{array}$ & \\
\hline & $\mathrm{n}=14$ & $\mathbf{n}=27$ & $P$ \\
\hline Age (years) ${ }^{\#}$ & $72(57-81)$ & $65(25-84)$ & 0.154 \\
\hline Gender (male/female) & $9 / 5$ & $17 / 9$ & 1.000 \\
\hline Difficulty index & $5(1-6)$ & $2.5(1-5)$ & 0.001 \\
\hline ASA-PS (3) & 0 & $4(14.8)$ & 0.278 \\
\hline Tumour type (HCC/metastatic tumour) & $3 / 11$ & $15 / 11$ & 0.103 \\
\hline Type of approach to the previous hepatectomy (Lap-Hx) & $6(42.9)$ & $8(29.6)$ & 0.501 \\
\hline Number of the previous hepatectomy ( 3 or more) & $4(28.6)$ & $2(7.4)$ & 0.159 \\
\hline Pringle manoeuvre (performed) & $9(64.3)$ & $8(29.6)$ & 0.102 \\
\hline Time of Pringle manoeuvre (minutes) $^{*}$ & $46(0-133)$ & $0(0-128)$ & 0.045 \\
\hline Year of surgery (2015-2018) & $14(100)$ & $14(51.9)$ & 0.003 \\
\hline Tumour diameter $(\mathrm{mm})^{\#}$ & $20(4-40)$ & $15(6-30)$ & 0.014 \\
\hline Tumour number (multiple) & $4(28.6)$ & $5(18.5)$ & 0.043 \\
\hline Tumour location $(\mathrm{S} 1, \mathrm{~S} 7$ or $\mathrm{S} 8)$ & $9(64.3)$ & $3(11.1)$ & 0.001 \\
\hline Tumours located in the ipsilateral of the primary site & $13(92.9)$ & $6(22.2)$ & $<0.001$ \\
\hline Intraoperative blood loss $(\mathrm{mL})^{*}$ & 284 (small amount-3,260) & 50 (small amount-784) & 0.009 \\
\hline Operation time (minutes) $^{\#}$ & $253(129-368)$ & $170(72-355)$ & 0.002 \\
\hline Converted to laparotomy & $2(14.3)$ & $2(7.4)$ & 0.602 \\
\hline Morbidity $\geq$ Clavien-Dindo grade $3 a$ & $1(7.1)$ & 0 & 0.350 \\
\hline Postoperative hospital stay (days) & $5(4-41)$ & $5(4-17)$ & 0.424 \\
\hline
\end{tabular}

Table 4. Comparisons of clinical factors of the patients who underwent LRH with and without tumours located in the adjacent area on the head or dorsal side of the primary site. LRH, laparoscopic repeat hepatectomy; ASA-PS, The American Society of Anesthesiologists physical status; HCC, hepatocellular carcinoma; Lap-Hx, laparoscopic hepatectomy; S, segment. Values in parentheses are percentages unless indicated otherwise; ${ }^{\#}$ value is expressed as the median (range).

a score $\geq 3$, as this factor resulted in a significant difference in intraoperative blood loss between our low- and intermediate-risk groups.

In the current study, we aimed to identify novel risk factors that predicted the difficulty of LRH, and we evaluated the recurrent tumour location using three methods. Our results showed that the presence of tumours located adjacent and cranial or dorsal to the previous surgical site, which was an independent risk factor for massive intraoperative blood loss in open repeat hepatectomy, was also a risk factor for blood loss in LRH, even though the incidence of this factor did not significantly differ between those who underwent LRH versus open repeat hepatectomy.

When comparing LRH and open repeat hepatectomy, we found that the selection of LRH or open hepatectomy depended on the presence of tumours located in segments 1, 7, or 8 and/or the presence of tumours ipsilateral to the previous surgical site, whereas the presence of tumours adjacent and cranial or dorsal to the previous surgical site was a more important factor when determining the difficulty of repeat hepatectomy. Therefore, if it is necessary to detach the surface adhesions resulting from previous liver resection, attention must be paid to the presence of tumours in segments other than S7 and/or S8, while LRH could be strongly considered for tumours located in segments 7 and/or 8 if adhesiolysis of the surface of the previous liver resection site is not necessary, as laparoscopy does not require the dissection of all adhesions, unlike in open surgery; adhesiolysis is performed only to access the operative field for hepatectomy in LRH.

When comparing the Iwate and novel difficulty scoring systems, the index for the low-risk group in the Iwate difficulty scoring system was almost the same in the novel difficulty scoring system, whereas the intermediate-risk group in the Iwate difficulty scoring system had a higher index in the novel difficulty scoring system. This suggests that there are many opportunities to detach surface adhesions at the previous liver resection site in cases with tumours located in segments 7 or 8 . Almost all patients with a score of more than 6 in the novel difficulty scoring system had tumours located in segments 7 or 8 and tumours cranial or dorsal to the previous surgical site.

The present study had several limitations, including the retrospective design, small sample size, and single centre setting. Although the number of patients undergoing LRH in our study was larger than in previous reports $^{6-10}$, further prospective multi-institutional studies are needed to validate our results. Another limitation is that we defined patients in the $\geq 75$ th percentile for blood loss volume as our high-risk group, and the results might differ depending on which factor and/or cut-off point is used to define high-risk patients among the several patient risk factors for LRH.

In conclusion, our results verified the usefulness of the Iwate difficulty scoring system to predict massive blood loss and identified the patient factors that predicted the difficulty of LRH. Our results suggest the usefulness of our novel difficulty scoring system for LRH based on the Iwate difficulty scoring system. 


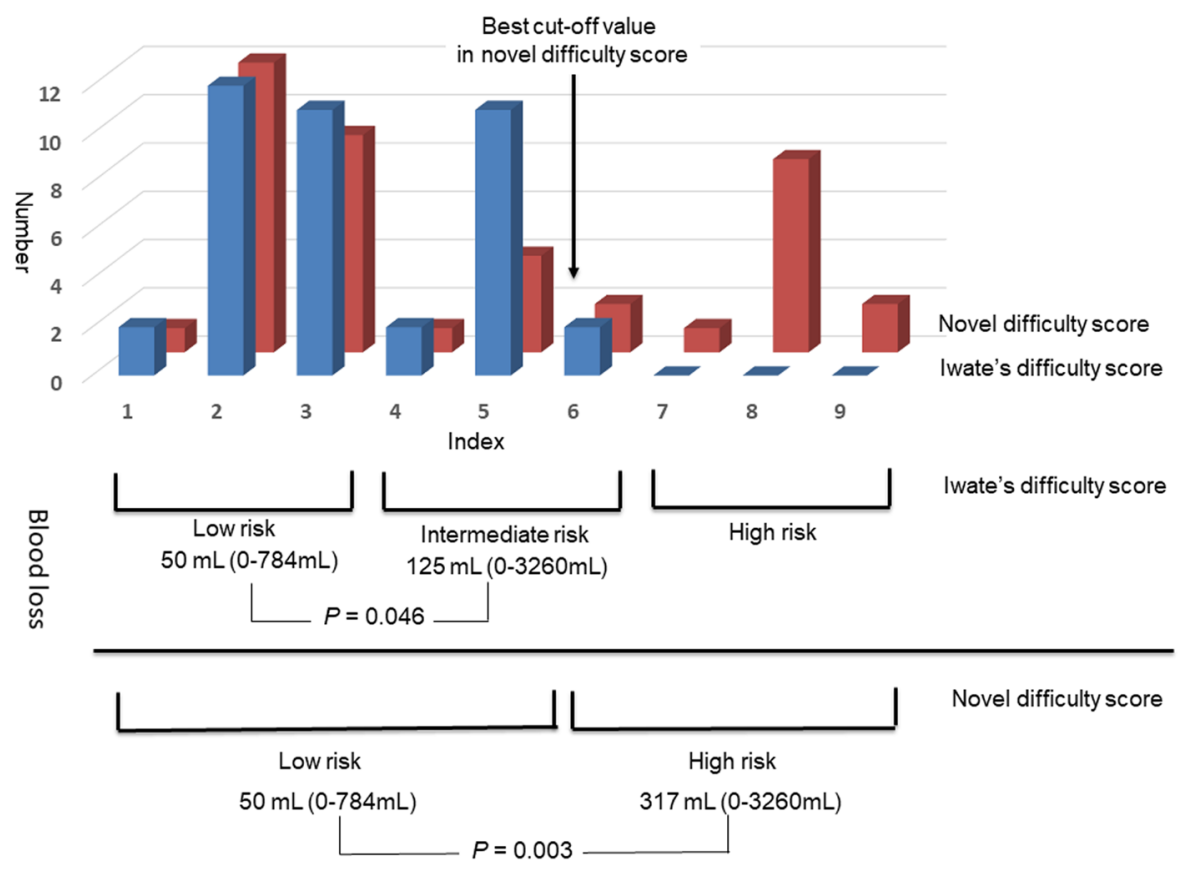

Figure 2. Distribution in accordance with the Iwate and novel difficulty scoring systems and the comparison of blood loss among risk groups classified in accordance with the Iwate and novel difficulty scoring systems.

\section{Methods}

Patients and methods. During the time period from the introduction of LLR in our institution in April 2011 until December 2018, 194 patients underwent repeat hepatectomy. Among these patients, we evaluated data from 137 patients who underwent partial hepatectomy or left lateral sectionectomy without thoracotomy; the laparoscopic approach was avoided for patients with recurrence who required anatomical resection except in those requiring left lateral sectionectomy or thoracotomy because of invasion to the diaphragm. We retrospectively reviewed the database of our institution from inception until January 2019. As this was a retrospective study, the Institutional Review Board of Shizuoka Cancer Center waived the need for patient consent (number: J2019-59-2019-1-3).

All included patients underwent preoperative abdominal ultrasonography, computed tomography, gadolinium ethoxybenzyl diethylene-triamine-pentaacetic-acid-enhanced magnetic resonance imaging, and laboratory assessment of liver function. We assessed liver function using the Child-Pugh classification ${ }^{16}$ and liver damage criteria $^{17}$ including the indocyanine green retention rate at 15 minutes. The indications for repeat hepatectomy were determined based on liver function and tumour location. Patients with early recurrence within 6 months were not candidates for repeat hepatectomy. Systemic chemotherapy was prescribed for patients with recurrent liver metastasis, while patients with recurrent HCC received non-surgical treatment such as transcatheter arterial chemoembolization, radiofrequency ablation, or sorafenib. The surgical procedure and extent of hepatectomy in each patient were determined during a weekly surgical conference.

We defined postoperative mortality as all in-hospital postoperative deaths, and classified complications into six grades in accordance with the modified Clavien-Dindo classification system ${ }^{18}$. When patients had undergone both laparoscopic and open previous hepatectomy, the approach to the previous hepatectomy was considered as open. We defined resection margins as R0 (tumour-free), R1 (microscopic tumour involvement), and R2 (macroscopic tumour involvement).

Surgical procedure in LRH. The first trocar for laparoscopy was inserted via a small laparotomy to avoid injuring the intra-abdominal organs affected by adhesions from the previous hepatectomy. After establishing pneumoperitoneum, three or four additional trocars were inserted. In most cases, it was possible to proceed with the operation, but sometimes one or two additional trocars were needed for adhesiolysis. Intraoperative ultrasonography was routinely performed to assess the tumours, understand the intrahepatic structures, and determine the transection line. The Pringle manoeuvre was routinely performed for as long as possible, even in repeat hepatectomy. However, the Pringle manoeuvre was abandoned when severe adhesions were present around the hepatoduodenal ligament. Hepatectomy was performed using an ultrasonic coagulation and incision system (HARMONIC ${ }^{\circledR}$ HD 1000i Laparoscopic Shears; Ethicon, Inc., Cincinnati, OH, USA) and a ball-type IO-advance electrode (VIO 300D; ERBE Elektromedizin, Tubingen, Germany).

Surgical procedure in open repeat hepatectomy. Open hepatectomy was routinely performed through a midline or a midline and subcostal incision, with thoracotomy added if needed. Hepatectomy was performed using an ultrasonic surgical aspirator (CUSA Excel; Integra LifeSciences, Plainsboro, NJ, USA) and an ultrasonic coagulation and incision apparatus chosen by the attending surgeon. 
A
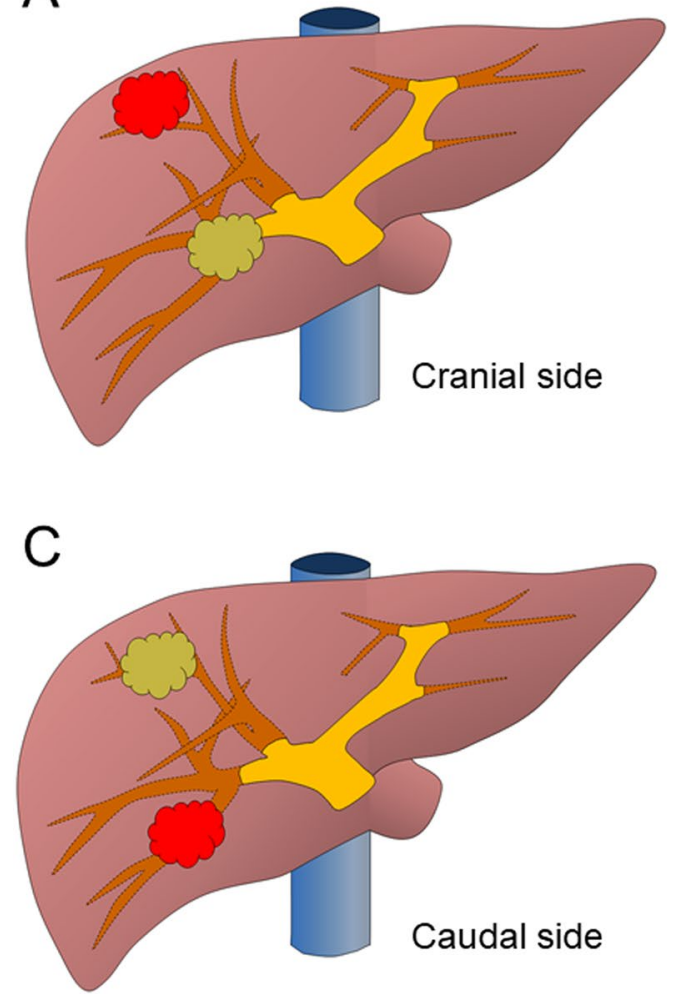
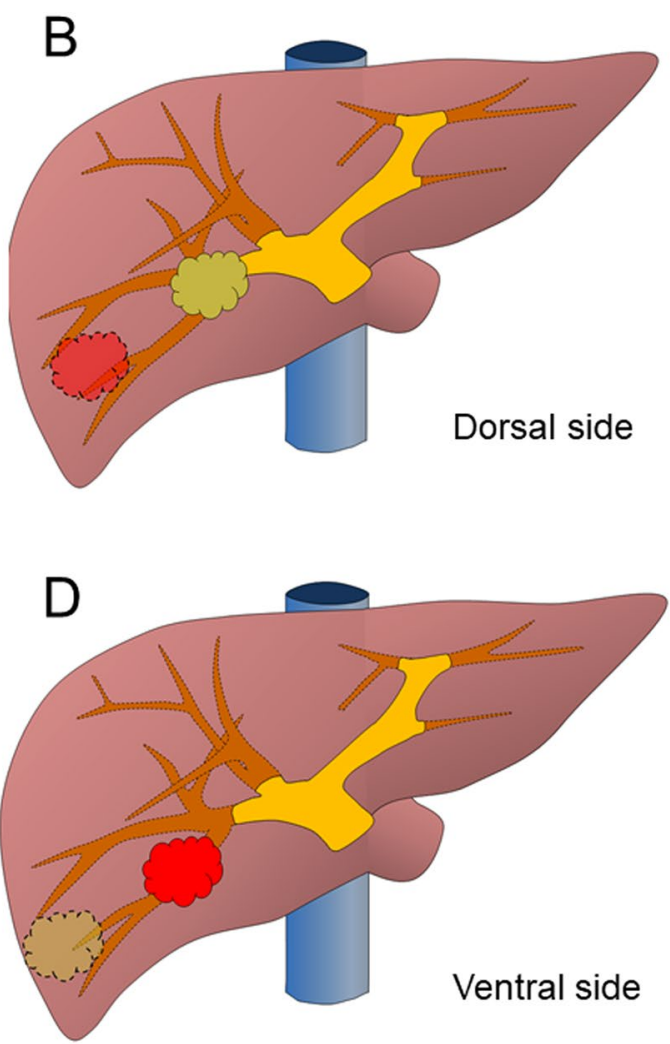

Figure 3. The positional relationship between the recurrent tumor and the previous tumor. The recurrent tumor is shown in red. (A) A recurrent tumor located adjacent and cranial to the previous surgical site. (B) A recurrent tumor located adjacent and dorsal to the previous surgical site. (C) A recurrent tumor located adjacent and caudal to the previous surgical site. (D) A recurrent tumor located adjacent and ventral to of the previous surgical site.

Validation of the usefulness of the difficulty scoring system proposed by Ban et al.11 in LRH. Patient clinical factors were compared between the low-, intermediate-, and high-risk groups classified in accordance with the difficulty scoring system of Ban et al. ${ }^{11}$

Recurrent tumour location relative to the previous surgical site. The recurrent tumour location was evaluated using three methods. First, we evaluated whether the recurrent tumour was located in segments 1,7 , or 8 , as it is difficult to perform LLR for these segments. Second, we evaluated whether the location of the recurrent tumour was ipsilateral to the previous surgical site, as adhesion dissection can be omitted if the recurrent tumour is located contralateral to the previous surgical site. Finally, we evaluated whether the recurrent tumour was located adjacent to the original site cranially (Fig. 3A) or dorsally (Fig. 3B), as adhesion dissection is also omitted if the recurrent tumour is located adjacent and caudal (Fig. 3C) or ventral (Fig. 3D) to the previous surgical site.

Definitions of the factors affecting massive blood loss in open repeat hepatectomy. In accordance with the difficulty scoring system for LLR ${ }^{11}$, blood loss volume and operation time were increased in the high-risk group. We defined the risk factors for massive intraoperative blood loss as those present in patients in the $\geq 75$ th percentile for blood loss volume in open repeat hepatectomy. We then examined whether these extracted risk factors were useful for risk assessment in LRH when combined with the Iwate difficulty scoring system $^{11}$.

Statistical analyses. Continuous variables are presented as the median and range and were compared using the Mann-Whitney U test. Categorical variables were compared using the chi-squared test or Fisher's exact test, as appropriate. The cut-off points for the laboratory data were defined as the upper limits of normal applied at our institution, while the cut-off points for age and tumour diameter were defined as the respective median values. Cumulative relapse-free and overall survival curves were analysed using the Kaplan-Meier method and compared using the log-rank test. Binomial logistic regression was used for the uni- and multivariate analyses, and all factors found to be significant predictors of massive blood loss in LRH $(P<0.05)$ in the univariate analysis were entered into the multivariate analysis. All statistical analyses were performed using the SPSS 24.0 software package (IBM Corp., Armonk, NY, USA), and two-tailed $P$ values of $<0.05$ were considered to indicate statistical significance. 
Ethical approval. This study confirmed to the ethical guidelines of the Declaration of Helsinki (2013 revision) and was retrospective in nature, and we obtained approval from the Institutional Review Board of Shizuoka Cancer Center (number: J2019-59-2019-1-3).

Received: 12 August 2019; Accepted: 1 November 2019; Published online: 27 November 2019

\section{References}

1. Mise, Y. et al. The Feasibility of Third or More Repeat Hepatectomy for Recurrent Hepatocellular Carcinoma. Ann Surg. 262, 347-357 (2015)

2. Yamashita, Y. et al. Third or more repeat hepatectomy for recurrent hepatocellular carcinoma. Surgery. 154, 1038-1045 (2013).

3. Hashimoto, M. et al. Efficacy of repeat hepatectomy for recurrence following curative hepatectomy for colorectal liver metastases: A Retrospective Cohort Study of 128 patients. Int J Surg. 36, 96-103 (2016)

4. Lee, H. et al. Repeat hepatic resection in patients with colorectal liver metastases. World J Gastroenterol. 21, 2124-2130 (2015).

5. Takahara, T. et al. Comparison of laparoscopic major hepatectomy with propensity score matched open cases from the National Clinical Database in Japan. J Hepatobiliary Pancreat Sci. 23, 721-734 (2016).

6. Shelat, V. G. et al. Outcomes of repeat laparoscopic liver resection compared to the primary resection. World J Surg. 38, 3175-3180 (2014).

7. Noda, T. et al. Laparoscopic Compared to Open Repeat Hepatectomy for Colorectal Liver Metastases: a Multi-institutional Propensity-Matched Analysis of Short- and Long-Term Outcomes. Surg Endosc. 32, 46-52 (2018).

8. Ome, Y. et al. The feasibility and efficacy of pure laparoscopic repeat hepatectomy. Surg Endosc. 32, 3474-3479 (2018).

9. Goh, B. K. P. et al. Perioperative Outcomes of Laparoscopic Repeat Liver Resection for Recurrent HCC: Comparison with Open Repeat Liver Resection for Recurrent HCC and Laparoscopic Resection for Primary HCC. World J Surg. 43, 878-885 (2019).

10. van der Poel, M. J. et al. Multicentre propensity score-matched study of laparoscopic versus open repeat liver resection for colorectal liver metastases. Br J Surg. https://doi.org/10.1002/bjs.11096 (2019). [Epub ahead of print].

11. Ban, D. et al. A novel difficulty scoring system for laparoscopic liver resection. J Hepatobiliary Pancreat Sci. 21, 745-753 (2014).

12. Uchida, H. et al. Laparoscopic liver resection in cirrhotic patients with specific reference to a difficulty scoring system. Langenbecks Arch Surg. 403, 371-377 (2018).

13. Tanaka, S. et al. Validation of a Difficulty Scoring System for Laparoscopic Liver Resection: A Multicenter Analysis by the Endoscopic Liver Surgery Study Group in Japan. J Am Coll Surg. 225, 249-258 (2017).

14. Uchida, H. et al. Clinical Utility of the Difficulty Scoring System for Predicting Surgical Time of Laparoscopic Liver Resection. J Laparoendosc Adv Surg Tech A. 26, 702-706 (2016).

15. Im, C. et al. Validation of difficulty scoring system for laparoscopic liver resection in patients who underwent laparoscopic left lateral sectionectomy. Surg Endosc. 31, 430-436 (2017).

16. Pugh, R. N., Murray-Lyon, I. M., Dawson, J. L., Pietroni, M. C. \& Williams, R. Transection of the esophagus for bleeding oesophageal varices. Br J Surg. 60, 646-649 (1973).

17. Liver Cancer Study Group of Japan General rules for the clinical and pathological study of primary liver cancer, 2008; 5th Japanese edition edn. Tokyo: Kanehara; (2008).

18. Dindo, D., Demartines, N. \& Clavien, P. A. Classification of surgical complications: a new proposal with evaluation in a cohort of 6336 patients and results of a survey. Ann Surg. 240, 205-213 (2004).

\section{Acknowledgements}

We thank Kelly Zammit, BVSc, from Edanz Editing (www.edanzediting.com/ac), for editing a draft of this manuscript.

\section{Author contributions}

Y.O.: Substantial contributions to the conception or design of the work, the acquisition, analysis and interpretation of data for the work and drafting the work. T.I., R.A., and K.O.: Acquisition of data for the work and revising it critically for important intellectual content. T.S. and Y.Y.: Interpretation of data for the work and revising it critically for important intellectual content. K.U.: Substantial contributions to the conception or design of the work, the acquisition and interpretation of data for the work, and revising it critically for important intellectual content. All authors gave final approval of the version to published and agree to be accountable for all aspects of the work in ensuring that questions related to the accuracy or integrity of any part of the work are appropriately investigated and resolved.

\section{Competing interests}

The authors declare no competing interests.

\section{Additional information}

Correspondence and requests for materials should be addressed to Y.O.

Reprints and permissions information is available at www.nature.com/reprints.

Publisher's note Springer Nature remains neutral with regard to jurisdictional claims in published maps and institutional affiliations.

Open Access This article is licensed under a Creative Commons Attribution 4.0 International format, as long as you give appropriate credit to the original author(s) and the source, provide a link to the Creative Commons license, and indicate if changes were made. The images or other third party material in this article are included in the article's Creative Commons license, unless indicated otherwise in a credit line to the material. If material is not included in the article's Creative Commons license and your intended use is not permitted by statutory regulation or exceeds the permitted use, you will need to obtain permission directly from the copyright holder. To view a copy of this license, visit http://creativecommons.org/licenses/by/4.0/.

(c) The Author(s) 2019 